\title{
Comparison of Measured and Estimated Resting Energy Expenditure in Adolescents and Young Adults With Severe Obesity Before and 1 Year After Sleeve Gastrectomy
}

Frances A. Rickard ${ }^{1,2}$, Landy P. Torre Flores ${ }^{2}$, Sonali Malhotra ${ }^{3}$, Alexander T. Toth ${ }^{2}$, Abeer Bader ${ }^{4}$, Fatima Cody Stanford ${ }^{2,4}$, Hang Lee ${ }^{5}$, Miriam A. Bredella ${ }^{6}$, Madhusmita Misra ${ }^{2,3}$ and Vibha Singhal ${ }^{2,3,4 *}$

${ }^{1}$ Melbourne School of Medicine, University of Melbourne, Melbourne, VIC, Australia, ${ }^{2}$ Neuroendocrine Unit, Massachusetts General Hospital and Harvard Medical School, Boston, MA, United States, ${ }^{3}$ Pediatric Endocrinology, Massachusetts General Hospital and Harvard Medical School, Boston, MA, United States, ${ }^{4}$ MGH Weight Center and Harvard Medical School,

OPEN ACCESS

Edited by:

Valentino Cherubini,

Azienda Ospedaliero Universitaria

Ospedali Riuniti, Italy

Reviewed by:

Ronald Cohen

University of Chicago, United States Gianpaolo De Filippo,

Bicêtre Hospital, France

${ }^{*}$ Correspondence: Vibha Singhal

vsinghal1@mgh.harvard.edu

Specialty section:

This article was submitted to

Pediatric Endocrinology,

a section of the journal

Frontiers in Pediatrics

Received: 03 December 2018

Accepted: 29 January 2019

Published: 19 February 2019

Citation:

Rickard FA, Torre Flores $L P$ Malhotra S, Toth AT, Bader A, Stanford FC, Lee H, Bredella MA, Misra M and Singhal V (2019)

Comparison of Measured and Estimated Resting Energy Expenditure in Adolescents and Young Adults With

Severe Obesity Before and 1 Year After Sleeve Gastrectomy.

Front. Pediatr. 7:37.

doi: 10.3389/fped.2019.00037
Boston, MA, United States, ${ }^{5}$ Biostatistics Unit, Massachusetts General Hospital and Harvard Medical School, Boston, MA, United States, ${ }^{6}$ Department of Radiology, Massachusetts General Hospital and Harvard Medical School, Boston, MA, United States

Introduction: Resting energy expenditure (REE) is often evaluated in adults and adolescents with obesity to estimate caloric requirements when advising dietary changes. However, data are lacking regarding the accuracy of methods used to clinically assess REE in adolescents with severe obesity. Moreover, there are no data regarding the effects of sleeve gastrectomy (SG) on REE in adolescents. We evaluated the accuracy and error rate between estimated and measured REE in adolescents with severe obesity and changes in REE following (SG).

Materials and Methods: Cross-sectional study (CSS): 64 adolescents and young adults, 14-22 years old, with moderate to severe obesity were enrolled. We measured REE (mREE) by indirect calorimetry and estimated REE (eREE) using Derumeaux (Deru), Mifflin-St Jeor (MS), Harris Benedict (HB), and World Health Organization (WHO) equations. DXA was used to determine body composition. Bland Altman analysis evaluated agreement between eREE and mREE. Longitudinal study: 12 subjects had repeat indirect calorimetry and DXA 1 year after SG. Longitudinal analysis was used to assess changes in REE and body composition.

Results: CSS: Median BMI was $45.2 \mathrm{~kg} / \mathrm{m}^{2}$ and median age was 18.0 (16.3-19.9) years. mREE correlated strongly with eREE . Bland Altman analysis demonstrated that only a few points were beyond the $1.96 \mathrm{SD}$ limit of disagreement. However, there was considerable overestimation of mREE by most equations. Longitudinal Study: In the subset that underwent SG, after 12-months, absolute REE decreased from 1709 $(1567.7-2234)$ to $1580.5(1326-1862.5)$ Calories $(p=0.002)$; however, the ratio of REE/Total Body Weight (TBW) increased from $13.5 \pm 2.3$ at baseline to $15.5 \pm 2.2$ at 1 year $(p=0.043)$. When evaluating parameters affecting $\%$ total weight loss, we 
found that it correlated positively with REE/TBW at 12 months $(R=0.625 ; p=0.03)$ and negatively with \% fat mass at 12 months $(R=-0.669 ; p=0.024)$.

Discussion: In adolescents with moderate-severe obesity, despite a correlation between mREE using indirect calorimetry and eREE using the Deru, MS, HB, and WHO equations, there is significant over-estimation of REE at the individual level, challenging their clinical utility. One year after SG, REE/TBW increased and strongly correlated with $\%$ total weight loss in adolescents.

Keywords: obesity, adolescents, resting energy expenditure, calorimetry, equation, sleeve gastrectomy

\section{INTRODUCTION}

Childhood and adolescent obesity is a growing public health concern. Currently, more than $20 \%$ of children in the United States have obesity, placing them at increased risk of obesity and its comorbidities as adults (1).

While obesity is a multifactorial disease, it is ultimately the result of a state of positive energy balance combined with a genetic predisposition for weight gain (2). To address energy imbalance, current recommendations for treating childhood obesity include family based lifestyle interventions with diet and exercise modifications (3). As part of this current strategy, energy requirements are often measured to guide the development of appropriate recommendations to achieve weight loss or maintenance. Indirect calorimetry is the current gold standard to measure resting energy expenditure (REE), but this technique is expensive and often difficult to access in clinical practice. Equations that estimate REE have been developed, however their accuracy in children with obesity is questionable, which makes it difficult to rely on these estimates in a clinical setting for this patient population (4).

Bariatric surgery is a useful treatment option for adolescents with severe obesity (5). When weight loss is achieved through diet and exercise, there is an associated decrease in REE $(6,7)$, which makes continued weight loss challenging. In adults, bariatric surgery results in decreases in absolute REE, but this decrease in REE is not evident when controlled for total body weight, suggesting that REE actually does not change (7). There are only a few studies in adults that have evaluated changes in REE after sleeve gastrectomy (SG), and results are conflicting $(8,9)$. To our knowledge, there are no studies that have assessed metabolic changes in adolescents after SG.

The primary objectives of this study are to (i) evaluate the accuracy of predictive equations used in clinics to estimate REE in adolescents with moderate to severe obesity, and (ii) to explore metabolic adaptations in adolescents and young adults with severe obesity 12 months following SG. We hypothesized that REE predictive equations would be inaccurate in adolescents with severe obesity, and that SG would lead to metabolic adaptations, causing a reduction in REE.

\section{METHODS}

This study was carried out in accordance with the Belmont principles and was approved by the Institutional Review
Board of Partners Healthcare System. All subjects gave written informed consent in accordance with the Declaration of Helsinki. Subjects $\geq 18$ years of age, and parents of subjects $<18$ years of age provided written informed consent, and informed assent was obtained from subjects $<18$ years of age. Sixty-four adolescents and young adults, ranging from 14-22 years of age, with moderate to severe obesity, BMI $>35 \mathrm{~kg} / \mathrm{m}^{2}$ were enrolled. Exclusion criteria included untreated thyroid dysfunction, smoking $>10$ cigarettes/day, substance abuse disorder, pregnancy or breast feeding as these effect REE. Four of our participants were on medications for attention deficit hyperactivity disorder (ADHD)-three were on dextroamphetamine and one on methylphenidate. Although, considered stimulant drugs, literature suggests that these medications do not alter REE (10). We did not exclude adolescents on stimulant medications given the frequency of their use in the general population, as this would limit the generalizability of our results. No participant was on stimulant medications for weight loss.

\section{MEASUREMENTS}

Indirect calorimetry (MetCart) was used to measure fasting REE under thermal neutrality using VMAX Encore 29 metabolic cart (Viasys Healthcare, Carefusion; San Diego, CA). All measurements were conducted at the Clinical Research Center of our institution. Subjects fasted for at least $8 \mathrm{~h}$ before measurement and were instructed to refrain from heavy exercise, tobacco, and alcohol the night before. Subjects did not alter their usual food intake the day prior to the visit. They rested for $20 \mathrm{~min}$ before measurement. Dual energy x-ray absorptiometry (Hologic QDR 4500) was used to measure body composition, specifically total fat and lean mass. Activity levels were recorded using the Paffenberger questionnaire.

Four REE equations were used to compare measured vs. estimated REE. These included the Derumeaux, Harris Benedict, World Health Organization/Food and Agriculture Organization and Mifflin- St Jeor equations (Table 1). These equations were chosen because clinically they are the most commonly used equations, and/or because younger populations were included in their initial derivation. Twelve subjects underwent SG and had repeat indirect calorimetry and DXA measurements 12 months after surgery. 
TABLE 1 | REE equations evaluated in adolescents with moderate-severe obesity.

\begin{tabular}{llll}
\hline Author & Sex & Age & Equations to estimate resting energy expenditure \\
\hline Derumeaux-Burel et al. (11) & Male & & $R E E=0.1096 \times F F M+2.8862$ \\
& Female & & $R E E=0.1371 \times F F M-0.1644 \times$ age +3.3647 \\
Harris et al. (12) & Male & & $R E E=66.473+(13.752 \times W)+(5.003 \times H)-(6.755 \times$ age $)$ \\
& Female & & $R E E=665.096+(9.5634 \times W)+(1.849 \times H)-(4.6756 \times a g e)$ \\
Mifflin et al. (13) & Male & & $R E E=(9.99 \times W)+(6.25 \times H)-(4.92 \times a g e)+5$ \\
& Female & & $R E E=(9.99 \times W)+(6.25 \times H)-(4.92 \times a g e)-161$ \\
MHO (14) & & $R E E=15.3 \times W+679$ \\
& Female & $<18$ & EE $=17.5 \times W+651$ \\
& & $R E E=15.3 \times W+496$
\end{tabular}

REE, resting energy expenditure; Deru, Derumeaux; WHO, World Health Origination; FFM, free fat mass; $\mathrm{kg}, \mathrm{W}$, weight, $\mathrm{kg} ; \mathrm{H}, \mathrm{height;} \mathrm{cm}$, age in years.

\section{STATISTICAL ANALYSIS}

JMP Pro12 (SAS Institute, Cary, NC, USA) was used for data analysis. The variables were assessed for normality of distribution and descriptive analysis is presented accordingly. The percentage of subjects with accurate prediction of REE on an individual level was also calculated, where an accurate prediction was defined as the estimated REE being between 90 and $110 \%$ of the measured REE (based on previous studies and clinical impact) (15). If the eREE was $<90 \%$ of mREE, this was defined as an under prediction, whereas if the eREE was $>110 \%$ of the MREE this was considered an over prediction. The percentage of the maximum positive/maximum negative error refers to the maximum positive and negative individual value of eREE that was the furthest away from the mREE (over and under prediction, respectively). Correlation is simple linear correlation between eREE and mREE. Bland Altman plots were used to evaluate the agreement between four predictive equations and measured REE (16).

For the longitudinal component of the study, we used the paired samples $t$-test or the Wilcoxon signed rank test, depending on distribution, to test if there was a significant within- group change over 12 months. For correlational analysis, Pearson's or Spearman's correlation was used depending on data distribution.

\section{RESULTS}

\section{Subject Characteristics}

The demographic and anthropometric characteristics of the 64 adolescents and young adults with moderate to severe obesity included in this study are displayed in Table 2. Almost half of our participants were Caucasians and $40 \%$ were Hispanic in ethnicity. The median hours of vigorous activity per week was $3.8(0-7)$.

\section{Accuracy of REE Predictive Equations}

Table 3 shows the difference between measured REE using indirect calorimetry and predicted REE using the four representative predictive equations (Table 1). The percentage of accuracy, over prediction, bias, maximum positive and negative error are listed. The Mifflin equation had the lowest difference from the mREE with a mean difference of $303.6 \mathrm{kcal} /$ day. The
TABLE 2 | Subject characteristics.

\begin{tabular}{lc}
\hline & $\boldsymbol{N}=\mathbf{6 4}$ \\
\hline Age, years & 18.0 (16.3-19.9) \\
Sex & 16 Male, 48 Female \\
RACE & \\
Caucasian (\%) & 48.4 \\
African American (\%) & 21.9 \\
More than one race (\%) & 12.5 \\
American Indian (\%) & 3.1 \\
Unknown (\%) & 12.5 \\
ETHNICITY & \\
Hispanic (\%) & 40.6 \\
Non-Hispanic (\%) & 59.4 \\
Weight, kg & $125.3(111.9-144.9)$ \\
Height, cm & $167.5 \pm 7.7$ \\
BMl, kg/m² & $45.2(40.4-49.0)$ \\
\% Ideal BMl for age & $209.6(190.1-229.8)$ \\
\% Fat Mass & $49.8(45.7-52.3)$ \\
\% Lean Mass & $48.8(46.8-53.1)$ \\
Vigorous exercise, h/wk & $3.8(0.0-7.0)$ \\
Sleeping hours, h/wk & $54.2 \pm 11.8$ \\
&
\end{tabular}

Values are presented as median (IQR) or mean $\pm S D$ or as a \% of total subjects. BMI, body mass index.

WHO equation was the furthest from the mREE, showing a mean difference $683.3 \mathrm{kcal} / \mathrm{day}$. Over all, the Mifflin equation performed best in our subjects with the lowest bias (18.9\%), and an accurate prediction of REE in $25 \%$ of subjects. The WHO equation performed the poorest showing the highest bias (39.3\%) and giving an accurate prediction in only $3.1 \%$ of subjects. The maximum positive error was estimated using the WHO equation, where one subject was predicted to have an REE that was $87.4 \%$ higher than the measured value. More than a third of the time, all four equations over predicted REE.

Bland Altman plots, Figure 1, shows the distributions of differences against the means, obtained with the two different methods, and the limits of agreement. Most subjects were within 
TABLE 3 | Comparison of measured REE and estimated REE in adolescents with moderate-severe obesity.

\begin{tabular}{|c|c|c|c|c|c|c|c|c|}
\hline $\begin{array}{l}\text { Predictive } \\
\text { equations }\end{array}$ & $\begin{array}{c}\text { Absolute } \\
\text { difference } \\
\text { (eREE -mREE) } \\
\text { (Kcal/day) }\end{array}$ & $\begin{array}{l}\text { Accurate } \\
\text { prediction } \\
(\%)\end{array}$ & $\begin{array}{c}\text { Under } \\
\text { prediction } \\
(\%)\end{array}$ & $\begin{array}{c}\text { Over } \\
\text { prediction } \\
(\%)\end{array}$ & $\begin{array}{l}\text { Bias } \\
(\%)\end{array}$ & $\begin{array}{c}\text { Maximum } \\
\text { negative error } \\
(\%)\end{array}$ & $\begin{array}{c}\text { Maximum } \\
\text { positive error } \\
(\%)\end{array}$ & $\begin{array}{c}\text { Correlation with } \\
\text { mREE }^{\star}\end{array}$ \\
\hline Deru & 480.7 & 14.1 & 1.6 & 84.4 & 27.9 & -13.5 & 62.2 & 0.70 \\
\hline $\mathrm{HB}$ & 435.3 & 9.4 & 1.6 & 89.1 & 25.6 & -15.5 & 70.8 & 0.78 \\
\hline Mifflin & 303.6 & 25 & 1.6 & 73.4 & 18.9 & -15.7 & 48.8 & 0.80 \\
\hline
\end{tabular}

Accurate prediction: percentage of patients predicted by equation within $10 \%$ of measured value.

Under prediction: percentage of patients predicted by this equation $<10 \%$ of measured value.

Over prediction: percentage of patients predicted by this equation $>10 \%$ of this measure value.

Bias: mean percentage error between predictive equation and measured value.

EREE, estimated resting energy expenditure; MREE, measured resting energy expenditure; Deru, Derumeaux; HB, Harris Benedict; WHO, World Health Organization. " $P$-value for all correlation $<0.0001$.
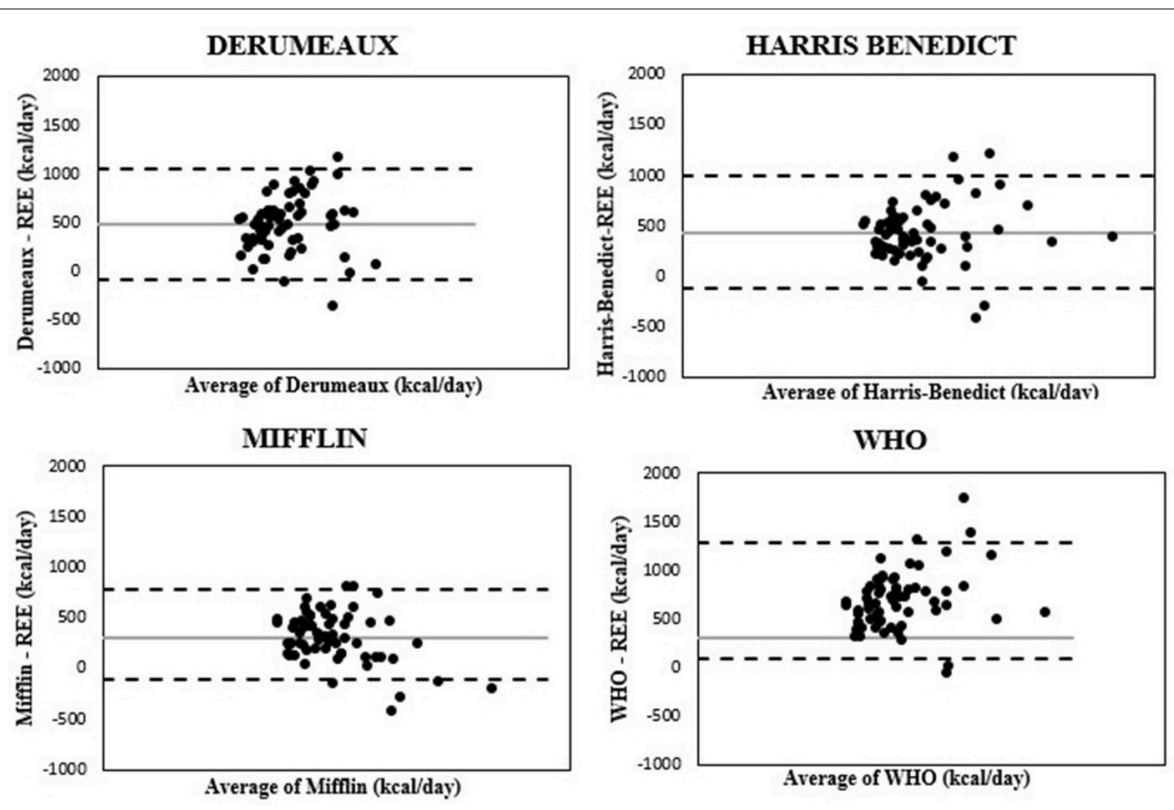

FIGURE 1 | Bland-Altman plots comparing absolute differences between measured and estimated REE and average of measured and estimated REE for the four equations. - , Mean; ---- , +/-1.96SD, •Subject.

the suggested limits of agreement across the four equations, and there was no evidence of dependency of the differences in the entire range of energy expenditure levels suggesting the lack of systematic error.

\section{REE and Metabolic Changes After Sleeve Gastrectomy}

Weight, body composition and metabolism changes from baseline to 12 months in adolescents and young adults undergoing SG $(N=12)$ are shown in Table 4 . The group was composed of mostly white (9/12) females. About $42 \%$ were of Hispanic ethnicity. The mean age baseline was $18.8 \pm 2.2$. As expected, they had a decrease in weight, BMI, total and $\%$ fat mass and total lean mass. Although the group showed a decrease in absolute REE, REE was unchanged after controlling for lean mass. Further, REE/total body weight increased 12 months after SG.

\section{Correlation of Weight Loss With Body Composition and REE After Sleeve Gastrectomy}

In an effort to seek the factors that could affect weight loss, we evaluated the correlation between total percent weight loss and body composition and REE parameters at baseline (before surgery) and after surgery. There was no correlation between percent total weight loss and body composition and REE parameters at baseline. However, percent total weight loss was positively associated with REE/TBW at 12 months and negatively with fat mass and \% fat mass at 12 months. This is shown in Table 5. 
TABLE 4 | Anthropometric and metabolic changes 1 year after sleeve gastrectomy.

\begin{tabular}{lccc}
\hline Characteristics & Pre-surgical & Post-surgical & $\boldsymbol{P}$-value \\
\hline Weight (kg) & $143.5 \pm 30.4$ & $100.1(90-115.5)$ & $\mathbf{0 . 0 0 0 5}$ \\
Excess body weight (\%) & $53.2 \pm 6.9$ & $41.4 \pm 13.7$ & $\mathbf{0 . 0 0 0 5}$ \\
BMl (kg/m²) & $50.1 \pm 8.6$ & $33.9(31-53)$ & $\mathbf{0 . 0 3 4}$ \\
Fat mass (kg) & $69.7 \pm 13.1$ & $44.9 \pm 11.5$ & $\mathbf{0 . 0 0 1}$ \\
\% Fat mass & $50.4 \pm 3.8$ & $43.7 \pm 6.1$ & $\mathbf{0 . 0 0 5}$ \\
Lean Mass (kg) & $65.7 \pm 9.6$ & $55.3 \pm 8.6$ & $\mathbf{0 . 0 0 1}$ \\
\% Lean mass & $47.8 \pm 3.6$ & $54.6 \pm 5.6$ & $\mathbf{0 . 0 0 3}$ \\
REE (kcal/day) & $1709(1567.7-2234)$ & $1580.5(1326-1862.5)$ & $\mathbf{0 . 0 0 2}$ \\
REE/LBM (kcal/day/kg) & $0.027(0.025-0.028)$ & $0.028 \pm 0.002$ & 0.577 \\
REE/TBW (kcal/day/kg) & $13.5 \pm 2.3$ & $15.5 \pm 2.2$ & $\mathbf{0 . 0 4 3}$
\end{tabular}

Values are presented as mean $\pm S D$ or as median (IQR). Excess body weight, weight ideal body weight (50th for age). BMI, body mass index; REE, resting energy expenditure; LBM, lean body mass; TBW, total body weight. Bold values indicate $p<0.05$.

\section{Correlation of Changes in Measured and Estimated REE Before and After Sleeve Gastrectomy}

To evaluate if the routinely used equations can at least be used to track the changes in REE before and after surgery, we ran a correlation analysis on the changes in REE obtained with indirect calorimetry ( $\mathrm{mREE}$ at 12 months $-\mathrm{mREE}$ at baseline $=\Delta \mathrm{mREE})$ with the changes in estimated REE obtained by each of the four equations (eREE at 12 months-eREE at baseline). We found that $\Delta$ mREE with $\Delta$ Mifflin eREE $(r=0.48 ; p=0.06) ; \Delta$ mREE with $\Delta$ Harris-Benedict eREE $(r=0.41 ; p=0.11) ; \Delta$ mREE with $\Delta$ WHO eREE $(r=0.52 ; p=0.04)$ and $\Delta$ mREE with $\Delta$ Deru eREE $(r=0.43 ; p=0.10)$.

\section{DISCUSSION}

\section{Resting Energy Expenditure Equations in Adolescents and Young Adults With Severe Obesity}

This study questions the utility of using predictive equations clinically to estimate REE in adolescents and young adults with moderate to severe obesity because of the high degree of variability in these predictions with a meaningful clinical error. Even the Mifflin equation, which provided the most reliable estimates, only accurately predicted REE in approximately one quarter of the subjects in our cohort. All four equations tended to overestimate REE with a high degree of bias (20-40\%) above the measured REE. The Bland-Altman plots, which quantify the agreement between the two quantitative measures, showed reasonable agreement for all the equations tested (16). While these agreements are acceptable at the population level, the degree of discrepancy at the individual level limits the clinical utility of using the equations in guiding weight management treatment. The average calorie deficit recommended to achieve a weight loss of 1-2 pounds/week is about $500 \mathrm{kcal} /$ day and most of these equations overestimated the individual calorie goal by that amount.
TABLE 5 | Correlation of \% total weight loss and body composition and measured REE before and after surgery.

\begin{tabular}{lccccc}
\hline & \multicolumn{2}{c}{ Pre-surgical } & & \multicolumn{2}{c}{ Post-surgical } \\
\cline { 2 - 3 } \cline { 5 - 6 } & r/Rho & $\boldsymbol{P}$-value & & r/Rho & P-value \\
\cline { 5 - 6 } PERCENT OF INITIAL WEIGHT LOST & & & & \\
\% Fat Mass & 0.003 & 0.992 & & -0.669 & $\mathbf{0 . 0 2 4}$ \\
Fat mass (kg) & 0.271 & 0.490 & & -0.642 & $\mathbf{0 . 0 3 3}$ \\
\% Lean Mass & -0.105 & 0.759 & & 0.576 & 0.064 \\
Lean Mass (kg) & 0.233 & 0.419 & & 0.032 & 0.926 \\
REE & -0.035 & 0.914 & & 0.028 & 0.931 \\
REE /LBM & -0.264 & 0.433 & & 0.436 & 0.178 \\
REE /TBW & -0.152 & 0.637 & & 0.625 & $\mathbf{0 . 0 3}$ \\
\hline
\end{tabular}

Linear Correlation- Spearman's test for non-parametric and Pearson for parametric distribution. BMI, body mass index; REE, resting energy expenditure; LBM, lean body mass; TBW, total body weight. Bold values indicate $p<0.05$.

Previous scientific literature has also found that equations are inaccurate in predicting REE for adolescents with obesity $(4,11,15,17)$. However, our results seem to have even lower accuracy when compared to other studies. Even when looking at the same equation, for example, Derumeaux, our study found an accuracy prediction in $14.1 \%$ of subjects, compared to Marra et al. (4), who found accurate predictions in $43 \%$ of females and $48 \%$ of males. Lazzer et al. (15) also tested the Derumeaux equation, finding an accurate prediction in $22 \%$ of subjects. The results of our study, and in the context of these other similar studies, suggest that predictive REE equations should be used with caution in this age and weight group.

Similarly, other research has reported inaccuracies between measured and predicted REE in adults with obesity (18). Marra et al. evaluated 1851 adults with obesity to find that overall prediction accuracy was low. Testing 15 different equations, an average of $55 \%$ of subjects were found to have an accurate predicted REE. When compared with our results, the accuracy of REE prediction in adolescents with obesity is far lower, reaching a mean $12.9 \%$ between the four equations.

There are different reasons for the lack of accuracy of these equations and these should be considered before applying them to patients. For example, in our study, the Harris Benedict equation accurately predicted REE in $6.3 \%$ of subjects, overestimating REE in the majority ( $91.7 \%$ of subjects). Harris and Benedict developed this equation using data from normalweight adults aged 20-70 in 1918, and the equation was not designed to predict REE in a younger population with excess weight. Though the Harris Benedict equation takes height, weight and age into account, other factors that contribute to REE such as fat free mass (11) and race (19) are not considered. In comparison, the Derumeaux equation includes fat free mass as a variable, but it was developed using data from children with obesity in a French population and does not take age into account for calculations in subjects. The ethnic diversity of our subjects may also contribute to the inaccuracy of this equation in predicting REE. Since REE can be affected by many factors at an individual level namely, lean mass, sleep, macronutrient composition of the previous meal and season 
of the year, it is extremely challenging to develop a precise equation at an individual level. Inaccuracy between measured REE and calculated REE has been reported in other studies of adolescents with obesity $(4,15,17)$, and given these results we suggest using caution while using these equations to predict REE in adolescents with obesity unless validated in the specific population being assessed.

\section{Energy Expenditure, Calorie Counting, and Weight Loss}

In clinical practice, an assessment of energy requirements has been a key component to guiding dietary recommendations for weight loss. The Academy of Nutrition and Dietetics, Intervention of the treatment of overweight and obesity in adults (20) suggest that the Mifflin equation should be used to estimate metabolic rate in adults, followed by a dietary intervention to decrease consumption of energy. The National Institute of Health $(\mathrm{NIH})$ suggests a deficit of $500-750 \mathrm{kcal} /$ day is required for weight loss. It provides a website and body weight planner based on the research by Hall et al. (21), with targeted deficits to achieve desired weight loss based on REE predictions. Eat for Health (22) is an initiative by the Australian Government that contains a similar calorie calculator. The initiative, in line with the Australian Dietary Guidelines and health promotion, suggests "any energy intake above the estimated requirement is likely to result in weight gain." Furthermore, many gym and fitness programs use predictive equations to guide their programs. Equinox, a worldwide luxury fitness company uses REE equations in their initial consultations to help individuals achieve their specified fitness goals. Popular phone application "My Fitness Pal", was founded in 2005 and has over 19 million monthly users. It aims to helps its users achieve their weight goals by asking them to input height, weight, gender and amount of desired weight loss, using predictive equations to give a daily net calorie goal as well as a specific date that your weight goal should be achieved. Weight Watchers uses a very similar approach, allocating an individual a number of "points" to eat per day based on the Mifflin equation. Textbooks such as Handbook of Clinical Nutrition and Dietetics (23) include whole chapters on predictive equations and how to use them.

New forms of technology including hand held indirect calorimeters and arm bands are being tested as a cheaper and more accessible option to measure REE accurately for continued use in the clinical setting $(24,25)$. On a different note, recent research suggests that perhaps the quality of calories ingested and not the quantity should be the primary focus for weight loss (26). In a study by Gardner et al. adult subjects with overweight and obesity were placed on a healthy diet with either low fat or low carbohydrate content and received instructional sessions every 2 weeks on healthy eating over 12 months, educating patients to eat more vegetables and less processed foods without following a caloric target. Subjects in both groups lost an average of 5-6 kilograms at 12 months regardless of diet in the absence of any caloric targets (26).

In addition to the inaccuracy of REE calculations, the accuracy of food labels, which are often used to estimate the caloric content, needs to be considered. Furthermore, the amount of calories extracted from any one food may differ from person to person. Studies have shown that our individual microbiome can affect digestion and the energy required to break down different foods (27). Thus, there are many variables that may impact the accuracy of calorie counting, leading to very crude estimations even in the hands of an astute patient.

The mental health effects of recommending calorie counting in adolescents should also be considered. Adolescents with obesity are at increased risk of developing an eating disorder and are more likely to use maladaptive behaviors to control weight (e.g., use of laxatives or induced vomiting) when compared to normal weight peers $(28,29)$. Students who diet by severely restricting their energy intake are at much greater risk of developing an eating disorder compared to those who do not diet (30).

Thus, given these inaccuracies in estimation of REE, compounded by the fact that it is difficult to get an accurate estimate of caloric intake and doing so may not be beneficial to the overall well-being of adolescents with obesity, we should challenge the utility of this practice. Providers, government recommendations and the health and fitness industry should move away from inaccurate energy expenditure calculations, calorie goals, and weight targets, and instead focus on promoting healthy lifestyle habits.

\section{Changes in Energy Expenditure After Bariatric Surgery}

Bariatric surgery is an effective weight loss tool (31), which results in long term weight loss and improvement in metabolic outcomes across the age spectrum (32). There are many factors that affect weight loss and weight regain after surgery, and alteration in REE is postulated to be one of these factors. Sleeve gastrectomy is currently the most commonly used bariatric procedure. To our knowledge, this is the first study that evaluates changes in REE after SG in adolscents (33). Our results show that absolute REE decreases after SG, consistent with reductions in total lean mass. When comparing these changes to a historical cohort where adults with obesity lost weight by lifestyle intervention (The Biggest Loser), we found a smaller reduction in REE in our cohort who underwent SG. After 7 months, Knuth et al. found that participants of the Biggest Loser lost $35 \pm 7.1 \%$ body weight, associated with a $617 \mathrm{kcal} /$ day reduction in REE (7). Interestingly, our cohort who underwent SG, lost a similar percentage of body weight to the biggest loser participants, however had a far lesser decrease in REE after 12 months. The smaller reduction in energy expenditure after SG or a "blunting" of adaptive metabolic change may be an additional mechanism to explain how SG is more effective as a weight loss strategy than conventional diet/exercise weight loss over time.

Despite a reduction in absolute REE, REE controlled for total body weight increases after SG in adolescents. This is likely a reflection of the relatively greater decrease in metabolically inactive fat mass compared to metabolically active lean mass. We also found a very strong correlation between percent total weight loss and the ratio of REE/TBW, suggesting that this 
may be a significant factor that affects the degree of weight loss in adolescents after SG as seen in adults after gastric bypass (9). Further monitoring is needed to determine whether this contributes to weight loss maintenance and if it can be an early predictor of response to surgery.

Moreover, when we evaluated the changes in REE as obtained by indirect calorimetry before and after surgery with the changes estimated using the equations before and after surgery in our small cohort, we did not find a strong correlation. This suggests that the predictive equations are not reliable to even monitor the trend after sleeve gastrectomy. This finding further emphasizes the guarded use of these equations in a surgical setting as well.

Limitations of this study include the small sample size. Due to limited numbers, we were unable to assess the accuracy of predictive REE equations in specific ethnic groups. We were unable to compare metabolic changes in bariatric surgery with adolescents with those who lost a comparable amount of weight with diet and exercise alone, given the practical difficulty of losing $20-30 \%$ of body weight by lifestyle measures.

\section{CONCLUSION}

Treating obesity remains a challenge. Predictive REE equations in adolescents with moderate to severe obesity have a considerable margin of error and should be used with caution in the clinical setting. It is time to shift away from calorie counting based on predictive estimates, given that these estimations are imprecise and may also be detrimental to the mental health of adolescents.

\section{REFERENCES}

1. Singh AS, Mulder C, Twisk JW, van Mechelen W, Chinapaw MJ. Tracking of childhood overweight into adulthood: a systematic review of the literature. Obes Rev. (2008). 9:474-88. doi: 10.1111/j.1467-789X.2008.00475.x

2. Kumar S, Kelly AS. Review of childhood obesity: from epidemiology, etiology, and comorbidities to clinical assessment and treatment. Mayo Clin Proc. (2017) 92:251-65. doi: 10.1016/j.mayocp.2016.09.017

3. Barlow SE, Expert Committee. Expert committee recommendations regarding the prevention, assessment, and treatment of child and overweight and obesity: summary report. Pediatrics (2007) 120 (Suppl. 4):S164-92. doi: 10.1542/peds.2007-2329C

4. Marra M, Montagnese C, Sammarco R, Amato V, Della Valle E, Franzese A, et al. Accuracy of predictive equations for estimating resting energy expenditure in obese adolescents. J Pediatr. (2015) 166:1390-6.e1. doi: 10.1016/j.jpeds.2015.03.013

5. Inge TH, Krebs NF, Garcia VF, Skelton JA, Guice KS, Strauss RS, et al. Bariatric surgery for severely overweight adolescents: concerns and recommendations. Pediatrics (2004) 114:217-23. doi: 10.1542/peds.114.1.217

6. Rosenbaum M, Leibel RL. Adaptive thermogenesis in humans. Int J Obes. (2010) (34 Suppl. 1):S47-55. doi: 10.1038/ijo.2010.184

7. Knuth ND, Johannsen DL, Tamboli RA, Marks-Shulman PA, Huizenga R, Chen KY., et al. Metabolic adaptation following massive weight loss is related to the degree of energy imbalance and changes in circulating leptin. Obesity (2014) 22:2563-9. doi: 10.1002/oby.20900

8. Bettini S, Bordigato E, Fabris R, Serra R, Dal Pra' C, Belligoli A. et al. Modifications of resting energy expenditure after sleeve gastrectomy. Obes Surg. (2018) 28:2481-6. doi: 10.1007/s11695-018-3190-3

9. Schneider J, Peterli R, Gass M, Slawik M, Peters T, Wölnerhanssen BK. Laparoscopic sleeve gastrectomy and Roux-en-Y gastric bypass lead to equal changes in body composition and energy metabolism 17 months
Instead, providers should focus on promoting a healthy lifestyle in the absence of caloric targets. SG, the most commonly used bariatric procedure in adolescents, is associated with increases in REE after controlling for total body weight, likely from the proportionally greater loss of metabolically inactive fat mass and maintenance of lean mass. These changes in REE also correlate with percent total weight loss 1-year post-surgery.

\section{AUTHOR CONTRIBUTIONS}

All authors have made substantial contribution to the paper. FR, LT, SM, AT, FS, and VS were responsible for acquisition of data and data analyses. FS, VS, and HL were responsible for interpreting the data. FR, LT, and VS were responsible for drafting the manuscript. VS was responsible for the conception and design of the study and critical review of the paper. $\mathrm{AB}, \mathrm{SM}, \mathrm{FS}, \mathrm{MB}$, $\mathrm{MM}$, and VS were responsible for revising the article critically and adding important intellectual content. $\mathrm{MB}, \mathrm{MM}$, and VS were responsible for acquiring the funding that sponsored the study. All authors have read and approved the final version of the paper.

\section{FUNDING}

NIH grant P30 DK040561 (FS, MB, MM, VS), NIH L30 DK118710 (FS), NIH R01 31 DK103946 (MB, MM), K24DK109940 (MB), K24HD071843 (MM), K23DK110419 (VS), and 32 P30DK040561 (VS). postoperatively: a prospective randomized trial. Surg Obes Relat Dis. (2016) 12:563-70. doi: 10.1016/j.soard.2015.07.002

10. Butte NF, Treuth MS, Voigt RG, Llorente AM, Heird WC. Stimulant medications decrease energy expenditure and physical activity in children with attention-deficit/hyperactivity disorder. J Pediatr. (1999) $135(2 \mathrm{Pt}$. 1):203-7. doi: 10.1016/S0022-3476(99)70023-5

11. Derumeaux-Burel H, Meyer M, Morin L, Boirie Y. Prediction of resting energy expenditure in a large population of obese children. Am J Clin Nutr. (2004) 80:1544-50. doi: 10.1093/ajcn/80.6.1544

12. Harris JA, Benedict FG. A biometric study of human basal metabolism. Proc Natl Acad Sci USA. (1918) 4:370-3.

13. Mifflin MD, St Jeor ST, Hill LA, Scott BJ, Daugherty SA, Koh YO. A new predictive equation for resting energy expenditure in healthy individuals. $A m$ J Clin Nutr. (1990) 51:241-7. doi: 10.1093/ajcn/51.2.241

14. WHO. Energy and protein requirements. Report of a joint FAO/WHO/UNU Expert Consultation. World Health Organ Tech Rep Ser (1985). p. 1-206.

15. Lazzer S, Agosti F, De Col A, Mornati D, Sartorio A. Comparison of predictive equations for resting energy expenditure in severely obese Caucasian children and adolescents. J Endocrinol Invest. (2007) 30:313-7. doi: $10.1007 / \mathrm{BF} 03346298$

16. Bland JM, Altman DG. Measurement error and correlation coefficients. BMJ (1996) 313:41-2. doi: 10.1136/bmj.313.7048.41

17. Acar-Tek N, Ağagündüz $D$, Çelik $B$, Bozbulut R. Estimation of resting energy expenditure: validation of previous and new predictive equations in obese children and adolescents. J Am Coll Nutr. (2017) 36:470-80. doi: 10.1080/07315724.2017.1320952

18. Marra M, Cioffi I, Sammarco R, Montagnese C, Naccarato M, Amato V., et al. Prediction and evaluation of resting energy expenditure in a large group of obese outpatients. Int J Obes. (2017) 41:697-705. doi: 10.1038/ijo.2017.34

19. Morrison JA, Alfaro MP, Khoury P, Thornton BB, Daniels SR. Determinants of resting energy expenditure in young black girls and young white 
girls. J Pediatrics (1996) 129:637-42. doi: $10.1016 /$ S0022-3476(96) 70142-7

20. Raynor HA, Champagne CM. Position of the academy of nutrition and dietetics: interventions for the treatment of overweight and obesity in adults. J Acad Nutr Diet (2016) 116:129-47. doi: 10.1016/j.jand.2015.10.031

21. Hall KD, Sacks G, Chandramohan D, Chow CC, Wang YC, Gortmaker SL., et al. Quantification of the effect of energy imbalance on bodyweight. Lancet (2011) 378:826-37. doi: 10.1016/S0140-6736(11)60812-X

22. Eat For Health. Available online at: https://www.eatforhealth.gov.au (2019) (Accessed January 3, 2019).

23. Stewart R. Handbook Of Clinical Nutrition And Dietetics. Southport: Griffith University, School of Public Health (2009).

24. Nieman DC, Austin MD, Chilcote SM, Benezra L. Validation of a new handheld device for measuring resting metabolic rate and oxygen consumption in children. Int J Sport Nutr Exer Metabol. (2005) 15:186-94. doi: 10.1123/ijsnem.15.2.186

25. Predieri B, Bruzzi P, Lami F, Vellani G, Malavolti M, Battistini NC. et al. Accuracy of sensewear Pro2 armband to predict resting energy expenditure in childhood obesity. Obesity (2013) 21:2465-70. doi: 10.1002/oby.20427

26. Gardner CD, Trepanowski JF, Del Gobbo LC, Hauser ME, Rigdon J, Ioannidis JPA., et al. Effect of low-fat vs low-carbohydrate diet on 12-month weight loss in overweight adults and the association with genotype pattern or insulin secretion: the DIETFITS randomized clinical trial. J Am Med Assoc. (2018) 319:667-79. doi: 10.1001/jama.2018.0245

27. Rowland I, Gibson G, Heinken A, Scott K, Swann J, Thiele I., et al. Gut microbiota functions: metabolism of nutrients and other food components. Eur J Nutr. (2018) 57:1-24. doi: 10.1007/s00394-017-1445-8

28. Sim LA, Lebow J, Billings M. Eating disorders in adolescents with a history of obesity. Pediatrics (2013) 132:e1026-30. doi: 10.1542/peds.2012-3940
29. Field AE, Camargo CA, Taylor CB, Berkey CS, Frazier AL, Gillman MW, et al. Overweight, weight concerns, and bulimic behaviors among girls and boys. $J$ Am Acad Child Adolesc Psychiatry (1999) 38:754-60.

30. Patton GC, Selzer R, Coffey C, Carlin JB, Wolfe R. Onset of adolescent eating disorders: population based cohort study over 3 years. BMJ (1999) 318:765-8. doi: $10.1136 / \mathrm{bmj} .318 .7186 .765$

31. Buchwald H, Avidor Y, Braunwald E, Jensen MD, Pories W, Fahrbach K., et al. Bariatric surgery: a systematic review and meta-analysis. J Am Med Assoc. (2004) 292:1724-37. doi: 10.1001/jama.292.14.1724

32. Inge TH, Jenkins TM, Xanthakos SA, Dixon JB, Daniels SR, Zeller MH., et al. Long-term outcomes of bariatric surgery in adolescents with severe obesity (FABS-5+): a prospective follow-up analysis. Lancet Diabetes Endocrinol. (2017) 5:165-173. doi: 10.1016/S2213-8587(16)30315-1

33. Griggs CL, Perez NP, Goldstone RN, Keller CM, Chang DC, Stanford FC, et al. National trends in the use of metabolic and bariatric surgery among pediatric patients with severe obesity. JAMA Pediatr. (2018) 72:1191. doi: 10.1001/jamapediatrics.2018.3030

Conflict of Interest Statement: The authors declare that the research was conducted in the absence of any commercial or financial relationships that could be construed as a potential conflict of interest.

Copyright (c) 2019 Rickard, Torre Flores, Malhotra, Toth, Bader, Stanford, Lee, Bredella, Misra and Singhal. This is an open-access article distributed under the terms of the Creative Commons Attribution License (CC BY). The use, distribution or reproduction in other forums is permitted, provided the original author(s) and the copyright owner(s) are credited and that the original publication in this journal is cited, in accordance with accepted academic practice. No use, distribution or reproduction is permitted which does not comply with these terms. 